\title{
PERFIL DOS CASOS NOTIFICADOS DE SÍFILIS CONGÊNITA
}

\author{
Kátia Fernanda Alves Moreira ${ }^{1}$, Davisson Michetti de Oliveira ${ }^{2}$, Lucas Noronha de Alencar ${ }^{2}$, Daniela Ferreira \\ Borba Cavalcante ${ }^{3}$, Aldrin de Sousa Pinheiro ${ }^{4}$, Nathalia Halax Orfão ${ }^{5}$
}

\begin{abstract}
RESUMO: Este estudo analisou a incidência de sífilis congênita, os exames para diagnóstico e tratamento fornecido aos recém-nascidos. Estudo epidemiológico descritivo, do tipo levantamento retrospectivo, com abordagem quantitativa. A coleta de dados foi realizada em dezembro de 2015 no Sistema de Informações e Agravos de Notificações, fornecido pelo Departamento de Vigilância Epidemiológica e Ambiental da Secretaria Municipal de Saúde de Porto Velho. A incidência de sífilis congênita foi crescente durante o período estudado (2009 a 2014) de 0,92 casos para 8,65/1.000 nascidos vivos. Em relação às gestantes, 157 (79,29\%) realizaram o prénatal, $119(60,10 \%)$ foram diagnosticadas durante o pré-natal e $109(55,1 \%)$ tratadas inadequadamente. Quanto aos recém-nascidos, 99 (50,00\%) eram do sexo feminino, 126 (63,64\%) pardas, 153 (77,27\%) nasceram assintomáticas e $129(65,15 \%)$ tratadas com penicilina G cristalina. Neste sentido, verificou-se que em Porto Velho há desafios quanto ao diagnóstico precoce e tratamento adequado das gestantes, parceiros e recém-nascidos.
\end{abstract}

DESCRITORES: Sífilis congênita; Cuidado pré-natal; Atenção primária à saúde; Saúde pública.

\section{PROFILE OF NOTIFIED CASES OF CONGENITAL SYPHILIS}

\begin{abstract}
The present study analyzed the incidence of congenital syphilis, tests for diagnosis, and treatment provided to newborns.This was a retrospective and descriptive epidemiological study with a quantitative approach.Data collection occurred in December 2015 using data from the Notifiable Diseases Information System provided by the Epidemiological and Environmental Surveillance Department of the Municipal Health Department of Porto Velho.The incidence of congenital syphilis increased during the period studied (2009 to 2014), from 0.92 cases to 8.65 per 1,000 live births.Regarding pregnant women, 157 (79.29\%) carried out prenatal care, $119(60.10 \%)$ were diagnosed during prenatal care, and $109(55.1 \%)$ underwent inappropriate treatment.With regard to newborns, $99(50.00 \%)$ were female, $126(63.64 \%)$ were brown, $153(77.27 \%)$ were born asymptomatic, and $129(65.15 \%)$ were treated with crystalline penicillin G.Therefore, there are challenges regarding early diagnosis and appropriate treatment of pregnant women, their partners, and newborns in the city of Porto Velho.

DESCRIPTORS: Syphilis, Congenital; Prenatal Care; Primary Health Care; Public Health.
\end{abstract}

\section{PERFIL DE CASOS NOTIFICADOS DE SÍFILIS CONGÉNITA}

RESUMEN: Se analizó la incidencia de sífilis congénita, los análisis para diagnóstico y tratamiento brindado a los recién nacidos. Estudio epidemiológico, descriptivo, tipo relevamiento retrospectivo, con abordaje cuantitativo. Datos recolectados en diciembre de 2015 del Sistema de Informaciones y Enfermedades de Notificación, ofrecido por el Departamento de Vigilancia Epidemiológica y Ambiental de la Secretaría Municipal de Salud de Porto Velho. La incidencia de sífilis congénita fue creciente durante el período estudiado (2009 a 2014), de 0,92 casos a 8,65/1.000 nacidos vivos. Respecto de las gestantes, 157 (79,29\%) realizaron el prenatal; 119 $(60,10 \%)$ fueron diagnosticadas durante el prenatal, y $109(55,1 \%)$ tratadas incorrectamente. En relación a los recién nacidos, 99 $(50,00 \%)$ eran de sexo femenino, $126(63,4 \%)$ mulatos, $153(77,27 \%)$ nacieron asintomáticos y $129(65,15 \%)$ tratados con penicilina G cristalina. Se verificó que en Porto Velho existen desafíos relativos al diagnóstico precoz y tratamiento adecuado de las gestantes, parejas y recién nacidos.

DESCRIPTORES: Sífilis Congénita; Atención Prenatal; Atención Primaria de Salud; Salud Pública.

${ }^{1}$ Enfermeira. Doutora em Enfermagem. Docente de Enfermagem da Fundação Universidade Federal de Rondônia. Porto Velho, RO, Brasil.

${ }^{2}$ Discente de Enfermagem. Fundação Universidade Federal de Rondônia. Porto Velho, RO, Brasil.

${ }^{3}$ Enfermeira. Mestra em Ensino em Ciências da Saúde. Docente de Enfermagem da Fundação Universidade Federal de Rondônia. Porto Velho, RO, Brasil.

${ }^{4}$ Enfermeiro. Mestre em Ensino em Ciências da Saúde. Docente de Enfermagem da Fundação Universidade Federal de Rondônia. Porto Velho, RO, Brasil.

${ }^{5}$ Enfermeira. Doutora em Enfermagem. Docente de Enfermagem da Fundação Universidade Federal de Rondônia. Porto Velho, RO, Brasil.

Autor Correspondente:

Recebido: $21 / 10 / 2016$

Davisson Michetti de Oliveira

Fundação Universidade Federal de Rondônia

Finalizado: $27 / 03 / 2017$

R. Algodoeiro, 4890 - 76.808-114 - Porto Velho, RO, Brasil

E-mail: davissomichetti@gmail.com 


\section{INTRODUÇÃO}

A sífilis é uma infecção sexualmente transmissível (IST) causada pela bactéria Treponema pallidum ${ }^{(1)}$. Quando esta infecção atinge gestantes que não realizam o tratamento ou realizam inadequadamente, esta doença pode ser transmitida para o concepto e recebe a denominação de sífilis congênita (SC) ${ }^{(2)}$. A transmissão vertical da sífilis pode ocorrer em qualquer período gestacional ou durante o parto ${ }^{(2-3)}$.

Desde 1986, a SC foi incluída entre as doenças de notificação compulsória no Brasil. Entretanto, quando observado o panorama nacional ao longo do tempo, percebe-se que pouco mudou, dado preocupante, uma vez que a sífilis é doença tratável/curável, com tratamento de baixo custo quando não há complicações ${ }^{(1,4)}$.

A Organização Pan-Americana da Saúde (OPAS) tinha como meta, até 2015, diminuir a incidência de SC para 0,5 caso por 1.000 nascidos vivos (NV), além de fornecer diagnóstico precoce e tratamento adequado para no mínimo $95 \%$ das gestantes e parceiros ${ }^{(5)}$.

Enxergando a SC como problema de saúde pública e a necessidade de alcançar as metas estabelecidas pela OPAS, o Ministério da Saúde criou em 2011 a estratégia "Rede Cegonha" com o objetivo de humanizar e assegurar o acompanhamento da gestante e criança durante o pré-natal, parto e puerpério no Sistema Único de Saúde (SUS). Desta maneira, a realização dos testes rápidos, entre eles o de sífilis, garantiria o diagnóstico precoce e tratamento da infecção ${ }^{(1-2,4)}$.

O diagnóstico de sífilis é realizado através do teste Venereal Disease Research Laboratory (VDRL) e/ ou teste rápido para sífilis durante a gestação, na primeira consulta de pré-natal e na $28^{a}$ semana ${ }^{(2)}$. Quando o VDRL é positivo, adota-se a conduta de tratamento da gestante e parceiro para prevenir que o concepto nasça com $\mathrm{SC}^{(1)}$.

O tratamento de sífilis pode ser ambulatorial ou hospitalar, a depender do estado geral da gestante. Para que seja considerado adequado, é necessário esquema medicamentoso e acompanhamento da gestante e parceiro; teste VDRL negativo ou diminuído, entre quatro a oito vezes em até seis meses e término do esquema farmacológico 30 dias antes do nascimento da criança ${ }^{(6)}$.

O tratamento é realizado preferencialmente com penicilina benzatina, por ser capaz de atravessar a barreira transplacentária e tratar mãe e feto ao mesmo tempo, com dose padrão de 2.400 UI distribuídos nos dois glúteos, por via intramuscular (IM). A quantidade de doses varia de acordo com o estadiamento da sífilis tanto na gestante quanto no parceiro ou em caso de profilaxia. O uso de ceftriaxona 1.000mg por via IM ou endovenosa é alternativa para gestantes e não gestantes, sendo sua aplicação diária por até 10 dias $^{(1-2,6)}$.

Diante do cenário epidemiológico da sífilis em todos os estados da federação, com falta de matéria prima para a produção de penicilina para suprir o desabastecimento internacional ${ }^{(7)}$, tratamento inadequado $^{(8)} \mathrm{e}$, consequentemente, resistência medicamentosa ${ }^{(9)}$, elevada taxa de transmissão vertical no país (6,5 casos de SC para cada 1.000 nascidos vivos $)^{(10)}$ e as graves repercussões de morbimortalidade por essa infecção, é de suma importância descrever o perfil epidemiológico dos casos de SC do município de Porto Velho, estado de Rondônia (RO), no período de 2009 a 2014, a fim de identificar os desafios ainda existentes para a interrupção da transmissibilidade.

\section{- MÉTODO}

Trata-se de estudo epidemiológico descritivo, do tipo levantamento retrospectivo. O estudo foi realizado no município de Porto Velho, capital do estado de Rondônia, localizado na Região Norte do Brasil.

A estrutura de atendimento e acompanhamento dos casos de SC, no município de Porto Velho, conta com 38 Unidades Básicas de Saúde (UBS), um Centro de Referência da Saúde da Mulher, além do Hospital Maternidade Municipal Mãe Esperança, que atende gestantes e parturientes de risco habitual, e o Hospital de Base Dr. Ary Pinheiro, que atende gestantes e parturientes de alto risco. 
A coleta de dados correspondeu aos casos de SC em recém-nascidos de mães que residiam e realizaram o pré-natal em Porto Velho, notificados no Sistema de Informações e Agravos de Notificações (SINAN), no período de 2009 a 2014, os quais foram fornecidos pelo Departamento de Vigilância Epidemiológica e Ambiental (DVEA) da Secretaria Municipal de Saúde de Porto Velho-RO (SEMUSA) em dezembro de 2015.

Foi realizado levantamento das variáveis socioculturais e clínicas de gestantes: idade categorizada com intervalos de 10 anos, raça/cor, escolaridade, ocupação, momento do diagnóstico de sífilis materna e tratamento da gestante e parceiros. De recém-nascidos, as variáveis foram: sexo, raça/cor, presença de sinais e sintomas, exames laboratoriais e de imagem, e esquema de tratamento. Foram excluídos os casos notificados em outras cidades, além daqueles que não residiam e não realizaram o pré-natal no município de Porto Velho.

Para o cálculo da estimativa da taxa de incidência de SC, tomou-se por numerador o número de crianças menores de um ano, abortos e natimortos notificados pelo SINAN/SEMUSA/Porto Velho dentre residentes no município de Porto Velho-RO com diagnóstico de SC; e por denominador, o número de nascidos vivos registrados pelo Sistema de Informação sobre Nascidos Vivos SINASC/ SEMUSA/Porto Velho para o ano em questão, multiplicado por mil. Os dados foram analisados por meio de estatística descritiva no Excel.

Trata-se de um subprojeto do projeto matriz intitulado: "Morbimortalidade em Porto Velho: população do entorno das usinas hidrelétricas de Santo Antônio e Jirau" aprovado pelo Comitê de Ética em Pesquisa da Fundação Universidade Federal de Rondônia (UNIR), conforme parecer $n^{\circ} 870.192$ de 29 de setembro de 2014.

\section{- RESULTADOS}

Em Rondônia, foram notificados 326 casos de SC entre os anos de 2009 a 2014, destes 198 residiam e realizaram o pré-natal no município estudado. Nota-se que, durante o período estudado, houve comportamento crescente de casos em todos os anos. Além disso, a incidência de Porto Velho foi praticamente o dobro do estado de Rondônia (Tabela 1).

Tabela 1 - Comparação entre a incidência, estadual e do município, de sífilis congênita por 1000 nascidos vivos de 2009 a 2014. Porto Velho, RO, Brasil, 2015

\begin{tabular}{lcccccc} 
Ano & \multicolumn{3}{c}{ Rondônia } & \multicolumn{3}{c}{ Porto Velho } \\
\cline { 2 - 7 } & NV* $^{*}$ & $\begin{array}{c}\text { Casos } \\
\text { Notificados }\end{array}$ & Incidência & NV* & $\begin{array}{c}\text { Casos } \\
\text { Notificados }\end{array}$ & Incidência \\
\hline 2009 & 26.083 & 13 & 0,5 & 7.649 & 7 & 0,92 \\
\hline 2010 & 25.835 & 25 & 0,97 & 8.089 & 9 & 1,11 \\
\hline 2011 & 27.658 & 52 & 1,88 & 8.742 & 32 & 3,66 \\
\hline 2012 & 26.513 & 51 & 1,92 & 8.941 & 29 & 3,24 \\
\hline 2013 & 27.097 & 69 & 2,55 & 8.927 & 42 & 4,7 \\
\hline 2014 & 27.555 & 116 & 4,21 & 9.129 & 79 & 8,65 \\
\hline Total & 160.741 & 326 & 2,03 & 51.477 & 198 & 3,85
\end{tabular}

*Nascidos Vivos

Fonte: SINAN/SEMUSA/2015

No ano de 2009, é possível verificar que a incidência estadual foi de 0,50 para cada $1.000 \mathrm{NV}$ e a do município de 0,92/1.000 NV. No ano de 2014 houve aumento significativo, sendo 4,21/1.000 NV no nível estadual e 8,65/1.000 NV no âmbito municipal.

Na Tabela 2, é possível observar as características sociais e assistenciais maternas dos casos notificados com SC. 
Tabela 2 - Distribuição das características maternas dos casos notificados de sífilis congênita entre 2009 a 2014. Porto Velho, RO, Brasil, 2015

\begin{tabular}{|c|c|c|}
\hline Características & $N=198$ & $\%$ \\
\hline \multicolumn{3}{|l|}{ Idade (em anos) } \\
\hline 10 a 19 & 55 & 27,78 \\
\hline 20 a 29 & 98 & 49,49 \\
\hline 30 a 39 & 35 & 17,68 \\
\hline 40 ou mais & 7 & 3,53 \\
\hline Ignorada & 3 & 1,52 \\
\hline \multicolumn{3}{|l|}{ Raça/Cor } \\
\hline Parda & 140 & 70,71 \\
\hline Branca & 18 & 9,09 \\
\hline Preta & 13 & 6,56 \\
\hline Ignorado & 27 & 13,64 \\
\hline \multicolumn{3}{|l|}{ Escolaridade } \\
\hline Ensino Fundamental & 107 & 54,04 \\
\hline Ensino Médio & 53 & 26,77 \\
\hline Ensino Superior & 3 & 1,51 \\
\hline Ignorada & 35 & 17,68 \\
\hline \multicolumn{3}{|l|}{ Ocupação } \\
\hline Do lar & 147 & 74,24 \\
\hline Estudante & 11 & 5,56 \\
\hline Autônoma & 4 & 2,02 \\
\hline Assalariadas & 25 & 12,62 \\
\hline Ignorado & 11 & 5,56 \\
\hline \multicolumn{3}{|l|}{ Realizou pré-natal } \\
\hline Sim & 157 & 79,29 \\
\hline Não & 31 & 15,66 \\
\hline Ignorado & 10 & 5,05 \\
\hline \multicolumn{3}{|c|}{ Momento do diagnóstico de sífilis materna } \\
\hline Durante o pré-natal & 119 & 60,1 \\
\hline No momento do parto/curetagem & 56 & 28,28 \\
\hline Após o parto & 19 & 9,6 \\
\hline Não realizado & 2 & 1,01 \\
\hline Ignorado & 2 & 1,01 \\
\hline \multicolumn{3}{|l|}{ Tratamento das gestantes } \\
\hline Adequado & 37 & 18,69 \\
\hline Inadequado & 109 & 55,05 \\
\hline Não realizado & 38 & 19,19 \\
\hline Ignorado & 14 & 7,07 \\
\hline \multicolumn{3}{|c|}{ Tratamento do(s) parceiros das gestantes } \\
\hline Sim & 49 & 24,75 \\
\hline Não & 119 & 60,1 \\
\hline Ignorado & 30 & 15,15 \\
\hline
\end{tabular}

Fonte: SINAN/SEMUSA/2015 
Na variável idade das mães, a mínima encontrada foi de 13 anos e máxima de 44 anos, com média de 23,97 anos. Para faixa etária, 98 mulheres (49,49\%) encontravam-se entre 20 e 29 anos, seguida por 55 mulheres (27,78\%) com idade entre 10 e 19 anos. Para variável raça/cor da pele, 140 (70,71\%) se autodeclararam pardas e em 27 (13,64\%) esta variável apresentava-se como ignorada.

Em escolaridade, 107 mulheres (54,04\%) tinham estudado até o ensino fundamental, seguido por $53(26,77 \%)$ com ensino médio. Na variável ocupação nota-se que 147 (79,80\%) não exerciam atividade remunerada e apenas 27 (14,64\%) com alguma atividade remunerada.

Dentre os 198 casos notificados, 157 (79,29\%) gestantes realizaram o pré-natal, entretanto não foi possível verificar em qual trimestre o diagnóstico foi dado, nem o número de consultas realizadas durante o pré-natal.

O tratamento materno foi considerado inadequado em 109 casos $(55,05 \%)$ e $37(18,69 \%)$ tiveram acesso ao tratamento adequado. Além disso, 119 parceiros $(60,10 \%)$ não realizaram o tratamento para sífilis e não foi obtido acesso quanto à classificação do tratamento, apenas se foi ou não realizado. $O$ diagnóstico de sífilis materna foi realizado durante o pré-natal em 119 casos (60,10\%), seguido por 56 $(28,28 \%)$ no momento do parto/curetagem.

A Tabela 3 mostra 99 casos de SC nas crianças do sexo feminino (50\%) seguido por $92(46,46 \%)$ do sexo masculino. Para raça/cor da pele, 126 crianças (63,64\%) eram pardas.

Tabela 3 - Distribuição das características sociais, assistenciais e diagnósticas relativas aos casos de crianças, notificados entre 2009-2014. Porto Velho, RO, Brasil, 2015 (continua)

\begin{tabular}{|c|c|c|}
\hline Características & $\mathbf{N}$ & $\%$ \\
\hline \multicolumn{3}{|l|}{ Sexo } \\
\hline Masculino & 92 & 46,46 \\
\hline Feminino & 99 & 50 \\
\hline Ignorado & 7 & 3,54 \\
\hline \multicolumn{3}{|l|}{ Raça/cor } \\
\hline Parda & 126 & 63,64 \\
\hline Branca & 32 & 16,16 \\
\hline Preta & 7 & 3,54 \\
\hline Ignorado & 33 & 16,67 \\
\hline \multicolumn{3}{|c|}{ Presença de sinais e sintomas } \\
\hline Assintomático & 153 & 77,27 \\
\hline Sintomático & 4 & 2,02 \\
\hline Não se aplica & 1 & 0,51 \\
\hline Ignorado & 40 & 20,2 \\
\hline \multicolumn{3}{|c|}{ Teste não treponêmico - Sangue periférico } \\
\hline Reagente & 161 & 81,31 \\
\hline Não reagente & 22 & 11,11 \\
\hline Não realizado & 7 & 3,54 \\
\hline Ignorado & 8 & 4,04 \\
\hline \multicolumn{3}{|c|}{ Teste não treponêmico - Líquor } \\
\hline Não reagente & 6 & 3,03 \\
\hline Não realizado & 157 & 79,29 \\
\hline Ignorado & 35 & 17,68 \\
\hline \multicolumn{3}{|c|}{ Radiografia de ossos longos } \\
\hline Com alteração & 2 & 1,01 \\
\hline Sem alteração & 98 & 49,49 \\
\hline Não realizado & 57 & 28,79 \\
\hline Ignorado & 41 & 20,71 \\
\hline
\end{tabular}




\begin{tabular}{|c|c|c|}
\hline \multicolumn{3}{|l|}{ Esquema de tratamento } \\
\hline Penicilina G cristalina 100.000 a $150.000 \mathrm{Ul} / \mathrm{Kg} / \mathrm{dia}$ - 10 dias & 129 & 65,15 \\
\hline Penicilina G procaína 50.000 Ul/Kg/dia - 10 dias & 5 & 2,53 \\
\hline Penicilina G benzatina $50.000 \mathrm{UI} / \mathrm{Kg} / \mathrm{dia}$ & 4 & 2,02 \\
\hline Outro esquema & 33 & 16,66 \\
\hline Não realizado & 9 & 4,55 \\
\hline Ignorado & 18 & 9,09 \\
\hline
\end{tabular}

Fonte: SINAN/SEMUSA/2015

Quanto aos aspectos clínicos, 153 casos (72,27\%) assintomáticos e 33 (20,20\%) ignorados. Nos quesitos de exames laboratoriais e imagem, o teste não treponêmico em sangue periférico foi reagente em 161 (81,31\%) casos. Não foi realizado o teste não treponêmico em líquor em 157 (79,29\%) dos casos. A radiografia de ossos longos apresentou-se sem alterações em 98 (49,49\%) e não foi realizada em 57 casos $(28,79 \%)$.

O esquema de tratamento mais utilizado foi Penicilina G Cristalina 100.000 a $150.000 \mathrm{UI} / \mathrm{Kg} /$ dia durante 10 dias em 129 indivíduos (65,15\%). Em 33 casos (16,67\%) houve esquema diferente do estipulado pela ficha de notificação, mas sem informação no banco de dados de qual conduta utilizada.

\section{- DISCUSSÃO}

A taxa de detecção de SC no município de Porto Velho mostrou comportamento crescente ao longo do período de estudo. Em nenhum dos cinco anos estudado o município conseguiu alcançar a incidência máxima de 0,5 casos para 1.000 NV como proposto pela OPAS. O aumento de incidência pode estar associado à melhoria da notificação e investigação dos casos, além de maior qualidade de detecção de sífilis gestacional no pré-natal(11).

Um dos possíveis motivos para o dobro na incidência da SC, no ano de 2014, pode ser explicado pela não disponibilidade de matéria prima para produção da penicilina, conforme divulgado em âmbito nacional para o tratamento de sífilis. Com isso, os estoques de penicilina diminuíram drasticamente, e muitos estados brasileiros ficaram sem nenhum frasco do fármaco para tratar as mães, parceiros e crianças $^{(6)}$.

Quanto à incidência crescente, também foi observado em estudo realizado no estado do Ceará, num espaço temporal de 10 anos, a notificação de SC crescente durante toda a década estudada. Houve 0,56 casos por 10.000 NV no ano de 2000 e 49,32 por 10.000 NV no ano de $2010^{(12)}$.

Estudos nacionais a respeito de SC mostram a maioria das gestantes na faixa etária de 20 a 29 anos, além de se autodeclararem pardas ${ }^{(13-15)}$, de maneira semelhante como ocorreu neste estudo. A SC não é uma doença que tem preferência por grupos populacionais, entretanto mulheres jovens estão mais propensas a se infectarem pelo Treponema pallidum devido ao comportamento social. Geralmente estas mulheres, conforme observado em dados de dois estudos regionais brasileiros, têm múltiplos parceiros e não utilizam preservativo durante o ato sexual(15-16). Quanto à raça/cor da pele, a parda segue o padrão nacional de população miscigenada, com a maioria composta por pessoas que se autodeclaram pardos ou negros ${ }^{(15,17)}$.

A baixa escolaridade de 107 mulheres (54,04\%) neste estudo torna-se desafio para a saúde pública, pois a compreensão adequada sobre a patologia, tratamento e prevenção é de suma importância para acompanhamento adequado das gestantes diagnosticadas com sífilis. Espera-se que quanto maior o nível de instrução da população melhor serão as atitudes tomadas para manter-se saudável ${ }^{(18)}$.

Estudo em Brasília, no Distrito Federal, avaliou 67 gestantes/puérperas com SC. Dentre elas, 64,2\% tinham apenas o ensino fundamental ${ }^{(19)}$, dado que corrobora aos encontrados nesta pesquisa.

Entre as mulheres com sífilis na gestação desta pesquisa, 158 (79,80\%) exerciam atividade laboral não 
remunerada, significativamente maior do que aquelas com atividade remunerada. Pode-se relacionar este elevado percentual à baixa escolaridade, pois quanto menor a escolaridade, menor as chances de conseguir um emprego com boa remuneração e melhor qualidade laboral ${ }^{(3)}$.

De maneira semelhante, pesquisa no município do Rio de Janeiro analisou a assistência pré-natal para prevenir a transmissão vertical do Treponema pallidum e encontrou que a maioria das mulheres $(63,17 \%)$ não desenvolvia nenhuma atividade remunerada ${ }^{(19)}$.

A respeito do pré-natal, afirma-se que é o momento da mulher ser acolhida para garantir gestação segura a ela e ao feto. Embora esta seja uma estratégia para diagnóstico precoce da sífilis materna e tratamento, em tempo oportuno, para prevenção da transmissão vertical para o recém-nascido, 157 gestantes $(79,29 \%)$ nesta pesquisa realizaram o pré-natal, $119(60,10 \%)$ foram diagnosticadas com sífilis gestacional e o tratamento só foi considerado adequado em 37 mulheres (18,69\%).

Entre os anos de 2008 e 2013, na cidade de Belém, estado do Pará, a maioria das mulheres (78,49\%) mães de crianças diagnosticadas com sífilis congênita, realizou o pré-natal ${ }^{(20)}$. Na cidade de Montes Claros, em estudo entre 2007 e 2013, o tratamento para sífilis na gestação das mães foi considerado inadequado ou incompleto em $64,8 \%$ dos $\operatorname{casos}^{(21)}$.

O tratamento de sífilis é considerado adequado quando utilizada a penicilina, que impede a transmissão vertical do Treponema pallidum, para tratar a gestante e o parceiro, bem como encerrar o esquema farmacológico 30 dias antes do parto e ter titulação de VDRL inferior de quatro a oito vezes entre três e seis meses ${ }^{(1)}$.

Considera-se tratamento inadequado aquele realizado com qualquer medicamento que não a penicilina; tratamento incompleto, mesmo tendo sido feito com penicilina; tratamento inadequado para a fase clínica da doença ou instituído no prazo de até 30 dias antes do parto; parceiro(s) sexual(is) com sífilis não tratado(s) ou tratado(s) inadequadamente ${ }^{(8)}$.

Em estudo de casos de SC, realizado no estado do Amazonas, 67,1\% dos parceiros de gestantes com sífilis gestacional não foram tratados durante o pré-natal(22), semelhante aos achados deste estudo. Para diminuir a incidência de sífilis materna, é fundamental tratar concomitantemente parceiro(s) da gestante, a fim de diminuir ou evitar as reinfecções.

Em pesquisa realizada em São Luís, no estado do Maranhão, 286 crianças nascidas com SC (78,8\%) eram pardas ${ }^{(23)}$, que também condiz com a população do estado de Rondônia autodeclarada como parda $(68,0 \%)^{(24)}$. Estudo realizado no Distrito Federal, identificou-se que 26 crianças nascidas com SC $(52 \%)$ eram do sexo masculino ${ }^{(19)}$, dado este corroborado com a população masculina residente em Rondônia $(50,4 \%)^{(24)}$.

Com relação às manifestações clínicas, 153 crianças $(77,27 \%)$ eram assintomáticas. Pesquisa na cidade de Porto Alegre avaliou a recorrência de sífilis durante a gestação e mostrou que 68,8\% de recém-nascidos eram assintomáticos ${ }^{(25)}$. Ao nascer, a maioria dos recém-nascidos são assintomáticos, entretanto podem surgir manifestações clínicas precoces em menores de dois anos, até manifestações tardias quando surgem após esta idade ${ }^{(11)}$.

Os principais sinais clínicos da SC são alterações hepáticas e esplênicas manifestados por hepatoesplenomegalia, alterações na coloração da pele como icterícia devido à anemia, problemas na conformação óssea, detectada por dentes deformados, elevação do arco palatino, fissura orofacial ou mandíbula curta, tíbia em lâmina de sabre, fronte olímpica. Outras alterações podem surgir nos pulmões, olhos, rim e sistema nervoso ${ }^{(25-26)}$.

Nesta pesquisa, o teste não treponêmico de sangue periférico, quando realizado, mostrou-se reagente em $161(81,31 \%)$ indivíduos; o teste de líquor não foi realizado em 157 (79,29\%) dos casos, por se tratar de exame mais invasivo e por já ter resultados com o exame de sangue periférico e as alterações em radiografia de ossos longos mostraram-se inalteradas em 98 indivíduos (49,49\%). Um estudo realizado em Montes Claros evidenciou que 27,9\% dos recém-nascidos apresentaram VDRL positivo, com relação aos demais exames complementares para investigação (radiografia de ossos longos, exame de líquor e hemograma) 42 crianças $(45,2 \%)$ não realizaram nenhum desses exames ${ }^{(21)}$, seja pela manifestação clínica assintomática ou tardia da SC ${ }^{(11,25)}$. 
No presente estudo 129 recém-nascidos (65,15\%) com SC foram tratados com Penicilina G cristalina 100.000 a $150.000 \mathrm{UI} / \mathrm{Kg} /$ dia por 10 dias, o que demonstra tratamento inadequado. A Secretaria de Vigilância em Saúde preconiza o tratamento realizado com penicilina G Cristalina $50.000 \mathrm{UI} / \mathrm{Kg} / \mathrm{dose} \mathrm{e}^{(2)}$. Um estudo mostra que 16 recém-nascidos com SC (32,00\%) foram tratados com penicilina G Benzatina, entretanto 18 crianças (36\%) não receberam nenhum tipo de tratamento. Além disso, tal atitude mostra falta de preparo da equipe de saúde para atender às recomendações estabelecidas pelo Ministério da Saúde ${ }^{(19)}$.

A limitação deste estudo foi o quantitativo de variáveis que apresentaram campos ignorados, fato este que dificulta uma análise mais refinada dos dados apresentados. O preenchimento completo das fichas de notificações é de extrema importância para que o município, estado e União possam conhecer o verdadeiro perfil socioepidemiológico da população e, a partir daí, planejar estratégias e ações a fim de garantir tratamento e prevenção adequados para a necessidade de cada população.

\section{CONCLUSÃO}

A análise dos dados de SC no município de Porto Velho encontrou possíveis problemas quanto ao diagnóstico precoce e tratamento adequado das gestantes e recém-nascidos. Apesar de o pré-natal ter sido realizado por grande parte das mulheres, o diagnóstico durante o pré-natal não se deu em mesma proporção, evidenciando falha no diagnóstico precoce dos casos de sífilis na gestação. Além disso, a maioria das mulheres e seus parceiros não foram tratados corretamente.

Outro fator preocupante foi a forma de tratamento dos casos de SC, com doses duas ou três vezes maiores do que o preconizado. Percebe-se que ainda há um longo caminho a ser percorrido para que as taxas de SC atinjam as preconizadas pela OPAS e Ministério da Saúde, entretanto, entende-se que a falta de matéria prima para produção de Penicilina contribuiu para o aumento significativos destes casos.

\section{- REFERÊNCIAS}

1. Ministério da Saúde (BR). Secretaria de Vigilância em Saúde. Departamento de DST, AIDS e Hepatites virais. Boletim epidemiológico: Sífilis. [Internet] Brasília: Ministério da Saúde; 2015 [acesso em 23 mar 2016]. Disponível: http://www.aids.gov.br/sites/default/files/anexos/publicacao/2015/57978/_p_boletim_sifilis_2015_fechado_ pdf_p__18327.pdf.

2. Ministério da Saúde (BR). Instituto Sírio-Libanês de Ensino e Pesquisa. Protocolos de atenção básica: Saúde das mulheres. [Internet] Brasília: Ministério da Saúde; 2016 [acesso em 23 mar 2016]. Disponível: http://189.28.128.100/ dab/docs/portaldab/publicacoes/protocolo_saude_mulher.pdf.

3. Domingues RMSM, Saraceni V, Hartz ZMA, Leal MC. Sífilis congênita: evento sentinela da qualidade da assistência pré-natal. Rev. Saúde Pública. [Internet] 2013;47(1) [acesso em 15 fev 2016]. Disponível: http://dx.doi. org/10.1590/S0034-89102013000100019.

4. Pavanatto A, Alves LMS. Programa de humanização no pré natal e nascimento: indicadores e práticas das enfermeiras. Rev Enferm UFSM. [Internet] 2014;4(4) [acesso em 15 fev 2016]. Disponível: http://dx.doi. org/10.5902/2179769211329.

5. Organização Pan-Americana da Saúde (OPAS). Plan estratégico regional de la Organización Panamericana de la Salud para el control de la infección por el VIH/SIDA y las infecciones de transmisión sexual (2006-2015) (resolução CD46.R15). $46^{\circ}$ Conselho Diretor, 57 $7^{\text {a }}$ sessão do Comitê Regional da OMS para as Américas; 26 a 30 de setembro de 2005. [Internet] Washington (DC) EUA. Washington (DC): OPS, 2005 [acesso em 23 mar 2016]. Disponível: http://www.paho.org/hq/index.php?option=com_docman\&task=doc_download\&gid=18529\&Itemi $\mathrm{d}=270$ \&lang=es.

6. Ministério da Saúde (BR). Secretaria de Vigilância em Saúde. Departamento de DST, AIDS e Hepatites virais. Nota informativa n 006/2016/GAB/DDAHV/SVS/MS. [Internet] Brasília: Ministério da Saúde; 2016 [acesso em 06 jun 2016]. Disponível: http://www.aids.gov.br/sites/default/files/anexos/legislacao/2016/58919/nota_informativa_ no006_importancia_e_urgencia_na_a_82765.pdf. 
7. Ministério da Saúde (BR). Secretaria de Vigilância em Saúde. Departamento de DST, Aids e Heptites Virais. Protocolo Clínico e Diretrizes Terapêuticas para Atenção Integral às Pessoas com Infecções Sexualmente Transmissíveis. [Internet] Brasília: Ministério da Saúde; 2015 [acesso em 06 fev 2017]. Disponível: http://www.aids. gov.br/sites/default/files/anexos/legislacao/2015/58575/nota_informativa_109_assinada_pdf_20349.pdf.

8. Ministério da Saúde (BR). Transmissão vertical do HIV e sífilis: estratégias para redução e eliminação. [Internet] Brasília: Ministério da Saúde; 2014 [acesso em 06 fev 2017]. Disponível: http://www.aids.gov.br/sites/default/files/ anexos/publicacao/2014/56610/folder_transmissao_vertical_hiv_sifilis_web_pd_60085.pdf.

9. Nações Unidas no Brasil. Resistência a remédios leva OMS a mudar diretrizes para tratamento de sífilis, clamídia e gonorreia. [Internet] 2016 [acesso em 06 fev 2017]. Disponível: https://nacoesunidas.org/resistencia-aremedios-leva-oms-a-mudar-diretrizes-para-tratamento-de-sifilis-clamidia-e-gonorreia/.

10. Ministério da Saúde (BR). Secretaria de Vigilância em Saúde. Boletim Epidemiológico. Sífilis 2016. [Internet] Brasília: Ministério da Saúde; 2016 [acesso em 06 fev 2017]. Disponível: http://www.aids.gov.br/sites/default/files/ anexos/publicacao/2016/59209/2016_030_sifilis_publicao2_pdf_51905.pdf.

11. Sonda EC, Richter FF, Boschett Gi, Casasola MP, Krumel CF, Machado CPH. Sífilis Congênita: uma revisão da literatura. Rev. Epidemiol Control Infect. [Internet] 2013;3(1) [acesso em 23 mar 2016]. Disponível: http://dx.doi. org/10.17058/reci.v3i1.3022.

12. da Costa CC, Freitas LV, Sousa DMN, de Oliveira LL, Chagas ACMA, Lopes MVO, Damasceno AKC. Sífilis congênita no Ceará: análise epidemiológica de uma década. Rev. esc. enferm. USP. [Internet] 2013;47(1) [acesso em 05 jun 2016]. Disponível: http://dx.doi.org/10.1590/S0080-62342013000100019.

13. Lima MG, dos Santos RFR, Barbosa GJA, Ribeiro GS. Incidência e fatores de risco para sífilis congênita em Belo Horizonte, Minas Gerais, 2001-2008. Ciênc saúde coletiva. [Internet] 2013;18(2) [acesso em 13 mai 2016]. Disponível: http://dx.doi.org/10.1590/S1413-81232013000200021.

14. Domingues RMSM, Szwarcwald CL, Souza Junior PRB, Leal MC. Prevalência de sífilis na gestação e testagem pré-natal: Estudo Nascer no Brasil. Rev. Saúde Pública. [Internet] 2014;48(5) [acesso em 08 jun 2016]. Disponível: http://dx.doi.org/10.1590/S0034-8910.2014048005114.

15. Nonato SM, Melo APS, Guimarães MDC. Sífilis na gestação e fatores associados à sífilis congênita em Belo Horizonte-MG, 2010-2013. Epidemiol. Serv. Saúde. [Internet] 2015;24(4) [acesso em 08 jun 2016]. Disponível: http://dx.doi.org/10.5123/S1679-49742015000400010.

16. Carvalho IS, de Brito RS. Sífilis congênita no Rio Grande do Norte: estudo descritivo do período 2007-2010. Epidemiol. Serv. Saúde. [Internet] 2014;23(2) [acesso em 17 abr 2016]. Disponível: http://dx.doi.org/10.5123/S167949742014000200010.

17. Chaves J, Bassani DCH, Ghignatti B, Derlan CB, Koepp J, Possuelo L. Sífilis congênita: análise de um hospital do interior do estado do RS. Revista da AMRIGS. [Internet] 2014;58(3) [acesso em 19 abr 2016]. Disponível: http:// www.amrigs.org.br/revista/58-03/003.pdf.

18. Mesquita KO, Lima GK, Filgueira AA, Flôr SMC, Freitas CASL, Linhares MSC, et al. Análise dos casos de sífilis congênita em Sobral, Ceará: contribuições para assistência pré-natal. DST-J Bras Doenças Sex Transm. [Internet] 2012;24(1) [acesso em 17 abr 2016]. Disponível: http://www.dst.uff.br/revista24-1-2012/7.Analise\%20dos\%20 Casos\%20de\%20Sifilis\%20Congenita.pdf.

19. Magalhães DMS, Kawaguchi IAL, Dias A, Calderon IMP. Sífilis materna e congênita: ainda um desafio. Cad. Saúde Pública. [Internet] 2013;29(6) [acesso em 06 jun 2016]. Disponível: http://dx.doi.org/10.1590/S0102311X2013000600008.

20. Pereira DAP, Maia BP, Seto IIC, Bichara CNC. Infecção congênita em pacientes matriculados em programa de referência materno infantil. Rev. Para. Med. [Internet] 2015;29(1) [acesso em 03 jun 2016]. Disponível: http:// saudepublica.bvs.br/pesquisa/resource/pt/lil-747241.

21. Lafetá KRG, Martelli Júnior H, Silveira MF, Paranaíba LMR. Sífilis materna e congênita, subnotificação e difícil controle. Rev. bras. epidemiol. [Internet] 2016;19(1) [acesso em 05 jun 2016]. Disponível: http://dx.doi. org/10.1590/1980-5497201600010006. 
22. Soeiro CMO, Miranda AE, Saraceni V, dos Santos MC, Talhari S, Ferreira LCL. Syphilis in pregnancy and congenital syphilis in Amazonas State, Brazil: an evaluation using database linkage. Cad. Saúde Pública. [Internet] 2014;30(4) [acesso em 28 mai 2016]. Disponível: http://dx.doi.org/10.1590/0102-311X00156312.

23. Rodrigues LS, Lima RHS, Costa LC, Batista RFL. Características das crianças nascidas com malformações congênitas no município de São Luís, Maranhão, 2002-2011. Epidemiol. Serv. Saúde. [Internet] 2014;23(2) [acesso em 03 jun 2016]. Disponível: http://dx.doi.org/10.5123/S1679-49742014000200011.

24. Instituto Brasileiro de Geografia e Estatística (IBGE). Uma análise das condições de vida da população brasileira 2016. Aspectos demográficos. [Internet] 2016 [acesso em 25 jan 2017]. Disponível: http://www.ibge.gov.br/home/ estatistica/populacao/condicaodevida/indicadoresminimos/sinteseindicsociais2016/default_tab_xls.shtm.

25. Hebmuller MG, Fiori HH, Lago EG. Gestações subsequentes em mulheres que tiveram sífilis na gestação. Ciênc. saúde coletiva. [Internet] 2015;20(9) [acesso em 27 mai 2016]. Disponível: http://dx.doi.org/10.1590/141381232015209.20332014.

26. Vallejo C, Cifuentes Y. Caracterización y seguimiento durante seis meses de una cohorte de reciénnacidoscon sífilis congénita. Biomédica. [Internet] 2016;36(1) [acesso em 02 jun 2016]. Disponível: http://dx.doi.org/10.7705/ biomedica.v36i1.2661. 